\title{
FDTD Analysis Fiber Optic SPR Biosensor for DNA Hybridization: A Numerical Demonstration with Graphene
}

\author{
Md. Muztahidul Islam¹, Md. Mohaiminul Islam ${ }^{1}$, Youshuf C. Shimul ${ }^{2}$, Azizur Rahman ${ }^{1}$, A. \\ Akib Ruhe $^{1}$, Mehedi Hassan ${ }^{1}$, Md. Biplob Hossain ${ }^{3 *}$ \\ 1. Department of Electrical and Electronic Engineering, Bangladesh Army University of Science \& Technology, \\ Natore, Bangladesh \\ 2. Department of Electrical and Electronic Engineering, Rajshahi University of Engineering \& Technology, \\ Rajshahi, Bangladesh \\ 3. Department of Electrical and Electronic Engineering, Jashore University of Science and Technology, Jashore, \\ Bangladesh \\ *biplobh.eee10@gmail.com
}

Received: 17 January 2019; Accepted: 11 February 2019; Available online: 1 April 2019

\begin{abstract}
This article illustrates a design and finite difference time domain (FDTD) method based on analysis of fiber optic surface plasmon resonance (SPR) biosensor for biomedical application especially for DNA-DNA hybridization. The fiber cladding at the middle portion is constructed with the proposed hybrid of gold (Au), graphene, and a sensing medium. This sensor can be recognized adsorption of DNA biomolecules onto sensing medium of PBS saline using attenuated total reflection (ATR) technique. The refractive index (RI) is varied owing to the adsorption of different concentration of biomolecules. Result states that the sensitivity with a monolayer of graphene will be improved up to $40 \%$ than bare graphene layer. Owing to increased adsorption capability of DNA molecules on graphene, sensitivity increases compared to the conventional gold thin film SPR biosensor. Numerical analysis shows that the variation of the SPR angle for mismatched DNA strands is quite negligible, whereas that for complementary DNA strands is considerable, which is essential for proper detection of DNA hybridization. Finally, the effect of Electric field distribution on inserting graphene layer is analyzed incorporating the FDTD technique by using Lumerical FDTD solution software.
\end{abstract}

Keywords: ATR; DNA Hybridization; FDTD method; Graphene; SPR.

\section{Introduction}

SPR spectroscopy is a label-free detection method which has become an extensively applied during the last three decades and accepted bio-analytical technique for real-time detection and monitoring of biomolecular binding events [1]. A surface plasmon (SP) can be deliberately of in general terms as an oscillation of electron on the surface of a conductor, typically a thin metal film [2]. SP generation is highly dependent on the optical properties of materials that are within the immediate vicinity of the metal film [3]. Under the compatible conditions light can be coupled into the SP mode and monitored as the analytical signal. Only light with a wave vector that closely be seems that of the SP mode will reveal any degree of coupling. The extent of light coupling will be directly adhering to the optical properties of the materials in contiguity with the metal film. Surface plasmon resonance concept has found tremendous applications in biosensors for simple compact, robust, high speed and high sensitive biomolecular detection [2]. SPR biosensor is used for DNA-DNA hybridization, protein-protein, protein-DNA, enzyme-substrate or inhibitor, receptor-drug, lipid membrane-protein, protein-polysaccharide, cell or virus-protein [2], [3] and also useful in providing information on non-covalent interactions of biomolecules and are applicable to investigations that involve protein-to-protein interactions or protein-to-small molecule interactions such as enzyme-substrate, antibody-antigen, protein-nucleic acids, and protein-polysaccharides [4-5].

As a fundamental part of fiber optic SPR biosensor, a fiber is coated with a thin metallic film (Au, Ag, or Al) to distinct the sensing medium and the dielectric [6-8]. Here the sensing layer is used to functionalize the gold film, which consequently improves the absorption of biomolecules [8]. An essential constituent of SPR sensing device is large surface area based absorbing material; recently developed 2D nanomaterial graphene has satisfied this requirement regarding this [9]. Graphene and graphene oxide facilitate biomolecule adsorption because of their large surface area and rich $\pi$ conjugation structure, making them suitable as dielectric top layers for SPR sensing [10]. Graphene has great fields of applications in advanced electronics and optoelectronics due to exclusive characteristics of high carrier mobility, high optical transparency, exceptional mechanical flexibility and strength 
[11], [12]. Recent studies have focused graphene based diverse applications in optoelectronic devices, including touch panels, ultrafast lasers, solar cells, optical modulators, photodetectors, and polarizers [13]-[16]. Graphene is being considered an ideal material for wideband, high sensitivity biosensors because of having high carrier mobility, short carrier lifetime, and wideband.

In this paper, graphene embedded on gold film fiber optic sensor is proposed, where phosphate buffer saline (PBS) solution as sensing dielectric medium that is used to provide better adsorption of single strand target or probe DNA (ss-DNA) biomolecules. This sensor is allowed to recognize hybridization event in proper orientation of adenine (A), thymine (T), guanine (G) and cytosine (C) according to Fig. 1 in ref. [1]. The refractive index (RI) changes nearby the graphene and gold (Au) owing to the immobilization of the DNA molecule. The RI change will cause to turn a change in the propagation constant of the SPR wave, which can be optically ascertained by ATR method [1]-[3]. The fiber optic SPR biosensor design and analysis approach are obtained by simulating the proposed sracture using Lumerical FDTD solution software. We used minimum reflectance vs SPR angle characteristic (Rmin $\sim \theta_{\text {SPR }}$ ) as detecting attributor. By using graphene, sensor sensitivity is enhanced to $1+0.4 \mathrm{~L}$ (where $\mathrm{L}$ is the total of graphene coatings) comparing with conventional SPR sensor in article ref. [1]. Finally, this enhanced sensitivity is discussed in terms of electric field distribution near the graphene interface.

\section{Methodology}

\subsection{Theoretical design structure of the proposed biosensor}

The proposed SPR biosensor is composed of four layers, the configuration of which is shown in Fig. 1. The operating wavelength is $633 \mathrm{~nm}$ considering that the optical nonlinearity can be enhanced at high frequency (low wavelength). Throughout the photon energy range, interband optical transitions occur because of graphene's band structure [11], [17]. As a result of the optical Kerr effect [11], notable variation occurs in the refractive index if the operating frequency is increased as the beam intensity is on the order of $1 \mathrm{GW} / \mathrm{cm}^{2}$. The theoretical results show that $633 \mathrm{~nm}$ is such a wavelength at which the overall sensitivity of the sensor is enhanced with the minimal possible Kerr effect [11]. For the optical fiber based SPR sensor, the fiber core has a diameter D of $50 \mu \mathrm{m}$ and a sensing region length $£$ of $5 \mathrm{~mm}$, where the cladding is removed. The unclad portion of the fiber is coated with a sheet of Au film with thickness $d_{2}$ of $50 \mathrm{~nm}$. Additionally, another coating of a graphene layer with thickness $d_{3}$ $\left(\mathrm{d}_{3}=\mathrm{L} \times 0.34 \mathrm{~nm}\right)$ is deposited, where $\mathrm{L}$ is the number of graphene layers. The first layer is fiber core having refractive index $(\mathrm{RI}) ; \mathrm{n}_{\mathrm{C}}$ of 1.451 , the second layer is $\mathrm{Au}$, deposited on the base of core having complex RI of $\mathrm{n}_{\mathrm{Au}}$ $=0.1726+3.4218 \mathrm{i}$. The third layer is a Graphene having high complex RI; $\mathrm{n}_{\mathrm{g}}$ of $3+1.149106 \mathrm{i}$ and the fourth layer is a PBS solution as sensing dielectric medium [17], which RI is changed according to the sensing characteristic. The graphene-based biosensor adsorbs ssDNA biomolecules and produce a native increase in the $\mathrm{RI}$ at the Graphene-Au surface [3]. Light with a wavelength of $633 \mathrm{~nm}$ is emitted from a monochromatic source, and the corresponding data are collected with a detector and computer.

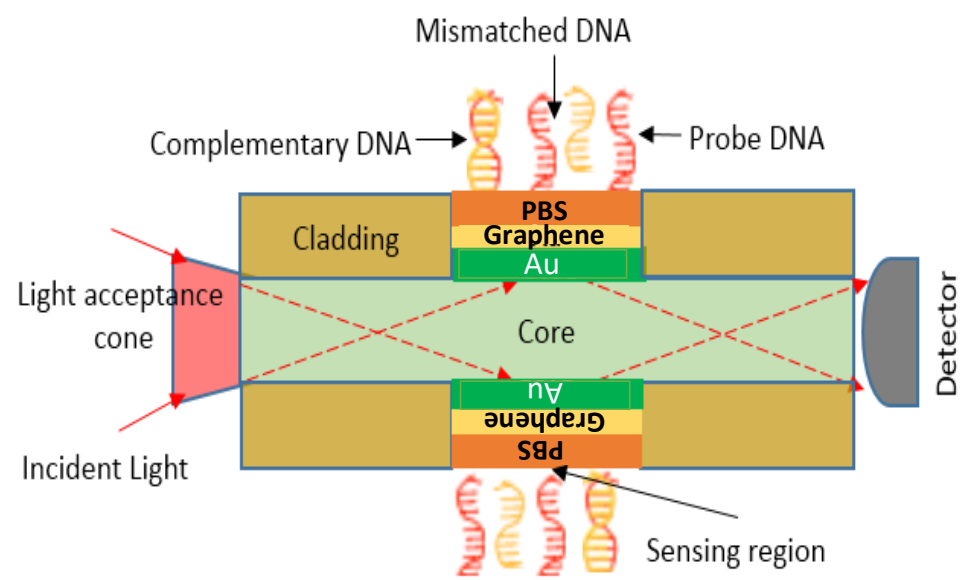

Fig 1. Schematic of 4-layered model for SPR biosensor: Core, Au (50 nm), graphene (0.34 nm) and sensing medium (PBS).

In DNA hybridization as depicted in Fig. 2, when two single-stranded (ssDNA) DNA, one is probe and the rest is target, is attached together and form double-stranded (dsDNA) helix structure, then it is defined as complementary hybridization event. The proposed model explains the analytical behavior of the sensor to detect the hybridization of target DNAs to the probe DNAs that are pre-immobilized on graphene. The SPR technology of this study also capable of distinguishing between complementary and single-based mismatched DNAs event. 


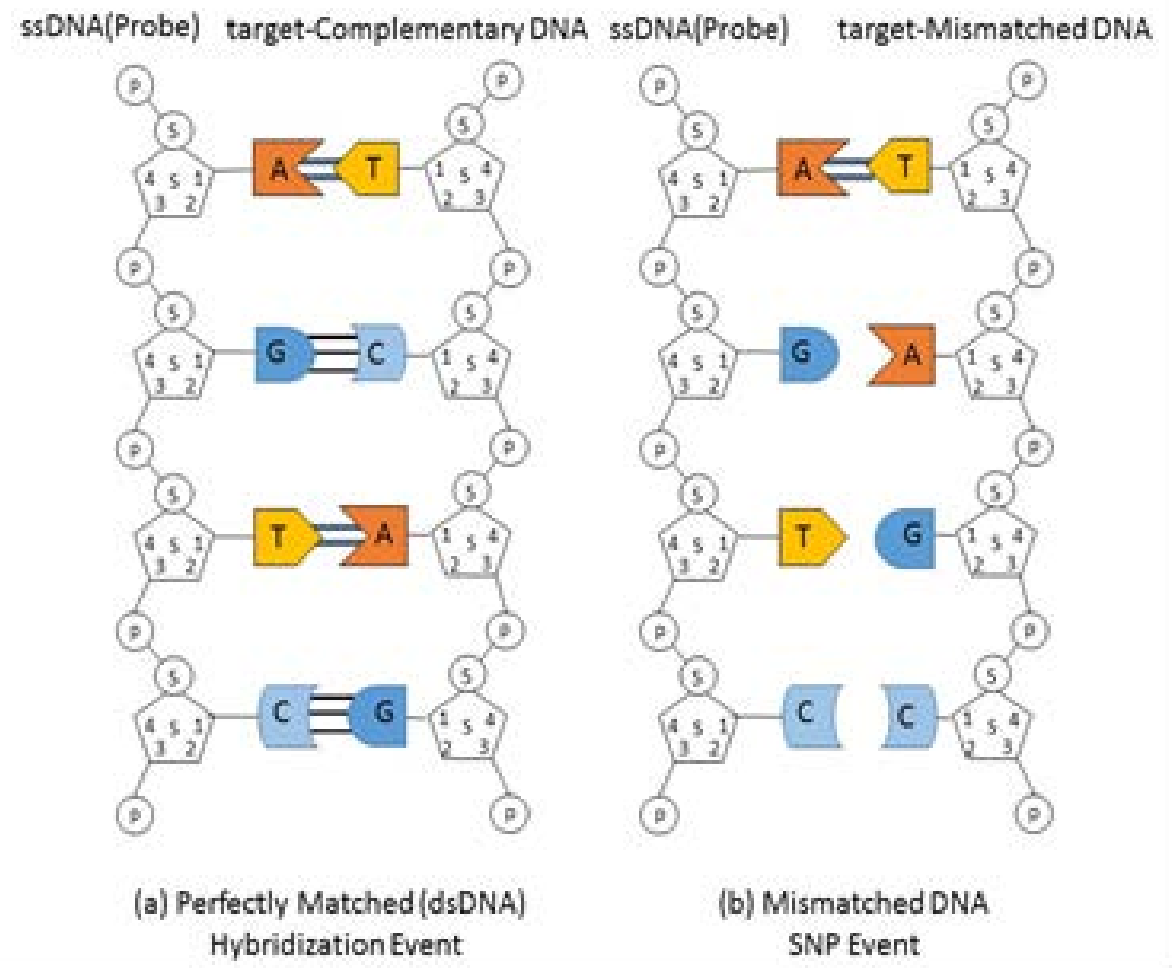

Fig 2. (a) Perfectly Matched ssDNA and Complementary DNA hybridization happened (Nitrogen base AdenineThymine (A-T) bond by di-hydrogen bonding, Guanine-Cytosine (G-C) bond by tri-hydrogen bonding) (b) Mismatched ssDNA and SNP happened.

\subsection{Mathematical modeling of the proposed SPR biosensor}

A TM polarized Gaussian modulated continuous wave with the center wavelength of $633 \mathrm{~nm}$ is used, which is generated an evanescent wave when passes through gold layer and is totally reflected at the gold interface. At this time evanescent wave propagates horizontally with propagation constant of following equation [1] [8]:

$$
k_{\text {in }}=2 \pi / \lambda_{633}\left(n_{c} \sin \theta_{\text {in }}\right)
$$

Equation (1) represents the ingredient of light propagating parallel to the gold surface. Here, $\theta_{\text {in }}$ is the angle of incidence of the light with the metal surface and $n_{c}$ is the RI of the core. The surface plasmon wave (SPW) propagation constant, $k_{s p w}$ is described in Equation (2), where $n_{A u}$ is the RI of the gold and $n_{g}$ is the RI of the graphene [1], [9]:

$$
k_{S P W}=\frac{2 \pi}{\lambda_{633}} \sqrt{\frac{n_{A u}^{2} n_{g}^{2}}{n_{A u}^{2}+n_{g}^{2}}}
$$

For TM polarization the electric field generated owing to the SPW along x-direction is specified in ref. [18] as:

$$
E_{x}=E_{0} \cos \theta_{i n} e^{\left(-j k_{i n} \cos \theta_{i n} z-j k \sin \theta_{i n} x\right)}
$$

Where $K_{\text {in }}$ is the propagation constant in layered media described in equation (1), $E_{0}$ is the magnitude of the electric field. The majority of SPR applications connect with the real RI changes due to chemical or biochemical action [1] and therefore, the imaginary components in Equation (3) will be neglected. The total reflectance vs angle of incidence or reflectance vs wavelength characteristics curve is known as the SPR curve. The SPR angle that can be represented in ref. [10], as:

$$
\theta_{S P R}=a \sin \sqrt{\frac{n_{A u}^{2} n_{S}^{2}}{n_{p}^{2}\left(n_{A u}^{2}+n_{S}^{2}\right)}}
$$


The shifting characteristics of the SPR angle owing to the variation of the refractive index are established as the following form [3]:

$$
n_{s}^{1}=n_{s}^{0}+c_{a} \frac{\Delta n}{\Delta c}
$$

Here, $n_{s}^{1}$ is the RI of the sensing dielectric (PBS) after adsorption of DNA molecules, $n_{s}{ }^{0}$ is RI of the PBS before adsorption of DNA molecules, $c_{a}$ is the concentration of adsorbed DNA molecules, and $\Delta n / \Delta c$ is the RI improvement due to the adsorbate DNA molecules. The RI improvement parameter ( $\mathrm{dn} / \mathrm{dc}$ ) is a value of $0.18 \pm$ $0.03 \mathrm{~cm}^{3} / \mathrm{g}$, irrespective of the identity of the protein and the buffer ions in the occasion of utilizing PBS buffer solution [19]. At SPR point, the propagation constant of incident wave represented by Equation (1) equals to the SPW represented by Equation (2). Change of the density (concentration) of sensing medium due to the immobilization of DNA causes the change of local RI $\left(n_{s}\right)$ of that sensing medium described by equation (5). The wavelength dependent refractive index of Au metal is calculated by the Drude-Lorentz model as:

$$
\mathrm{n}_{\mathrm{Au}}(\lambda)=\sqrt{1-\frac{\lambda^{2} \lambda_{c}}{\lambda^{2} p\left(\lambda_{c}+i \lambda\right)}}
$$

where the plasma wavelength, $\lambda \mathrm{p}$, is $\left(1.6826 \times 10^{-7} \mathrm{~m}\right)$ and the collision wavelength $\lambda \mathrm{c}$ is $\left(8.9342 \times 10^{-6} \mathrm{~m}\right)$, respectively [11].

The reflection intensity for TM-polarized light is expressed as [11]:

$$
\mathrm{Rp}=\left|\mathrm{r}_{\mathrm{p}}\right|^{2}
$$

Where

$$
r_{p}=\frac{\left(F_{11}+F_{12} g_{N}\right) g_{1}-\left(F_{21}+F_{22} g_{N}\right)}{\left(F_{11}+F_{12} g_{N}\right) g_{1}+\left(F_{21}+F_{22} g_{N}\right)}
$$

In equation (8), $\mathrm{F}$ is defined as

$$
F_{i j}=\left[\prod_{k=2}^{N-1} F_{k}\right]_{i j}=\left[\begin{array}{ll}
F_{11} & F_{12} \\
F_{21} & F_{22}
\end{array}\right]
$$

Here,

$$
F_{k}=\left[\begin{array}{cc}
\cos \beta_{k} & -\frac{i \sin \beta_{k}}{g_{k}} \\
-i g_{k} \sin \beta_{k} & \cos \beta_{k}
\end{array}\right]
$$

Here, $g_{\mathrm{k}}$ and $\beta_{\mathrm{k}}$ are expressed as:

$$
\begin{aligned}
& g_{k}=\left[\frac{\mu_{k}}{\varepsilon_{k}}\right]^{1 / 2} \cos \theta_{k}=\sqrt{\frac{\varepsilon_{k}-\left(n_{1} \sin \theta\right)^{2}}{\varepsilon_{k}^{2}}} \\
& \beta_{k}=\frac{2 \pi}{\lambda} n_{k} \cos \theta_{k}\left(z_{k}-{ }_{k-1}\right)=\frac{2 \pi}{\lambda} d_{k} \sqrt{\varepsilon_{k}-\left(n_{1} \sin \theta\right)^{2}}
\end{aligned}
$$

\section{Numerical results}

In this study, the finite difference time domain (FDTD) technique is used for analyzing the detection of bimolecular interaction by using commercial software Lumerical FDTD. The FDTD is a powerful method to solve Maxwell's equations in a nano film layer $(\mathrm{Au}-50 \mathrm{~nm})$ by using the YEE-algorithms. The FDTD method is a more reliable than others, such as multiple-multiple or Green's dynamic method in solving Maxwell's equations for complex geometries and dispersive media, such as gold and silver [13], [14]. The simulation was carried out with the Gaussian-modulated continuous wave with the center wavelength of $633 \mathrm{~nm}$. The perfectly matched layer (PML) boundary condition was used in such a way that waves enter into the layers with generating minimum reflections $\left(R_{\min }\right)$. The reflected intensity was reported using DFT reflectance and transmission monitor at 200nm away from Au/graphene interface. Graphene will adsorb the single strand DNA biomolecule (i.e. ssDNA) present in the sensing dielectric, and produce a local increase in the RI at the graphene surface. For the SPR curve, at resonant condition, the SPP is known as minimum total reflectance (i.e., ATR minimum). The angle of incidence 
at which ATR minimum is called SPR angle and this value is reported $57.61^{\circ}$ while inserting $1000 \mathrm{nM}$ concentrated perfectly matched target DNA molecule and also and also the minimum reflectance is 0.00105 reported shown in Fig 3.

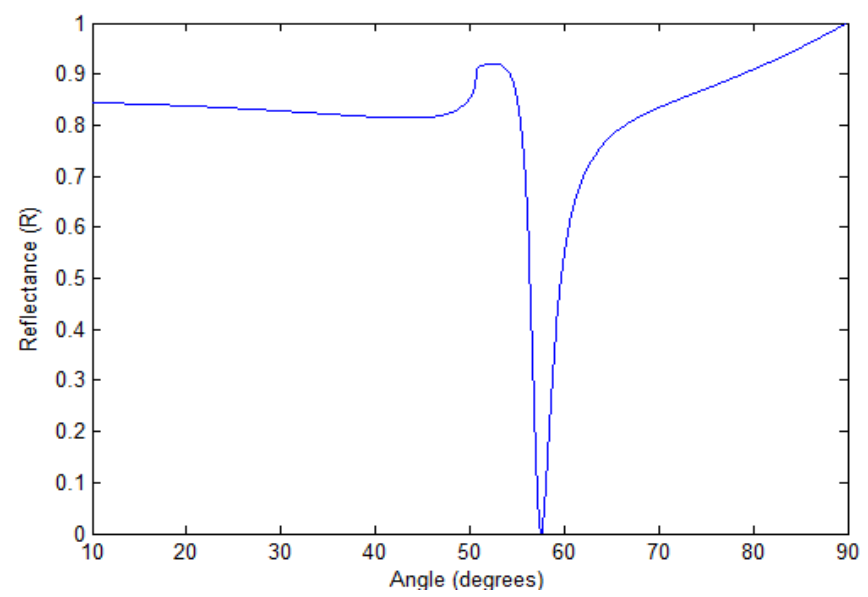

Fig 3. Numerical simulation for SPR curves (Reflectance versus SPR angle).

When the value of detecting attributor (SPR angle ) is greater than or equal to $57.61^{0}$ then complementary hybridization of DNA has been sensed and if the change of detecting attributor is smaller than that no consistencies consequence has been recognized and the recognition technique should be resumed.

The absorption improvement effects on addition of graphene layer is investigated by the means of the plasmon field distribution. For TM polarization, the electric field distribution owing to the SPW along the perpendicular to the core/Au interface is shown in Fig. 4. We stated the electric field distribution of the proposed biosensor structure with 10 graphene layers. In Fig. 4(a) and 4(b), the electric field distribution and the normalized electric field intensity have been obtained respectively.

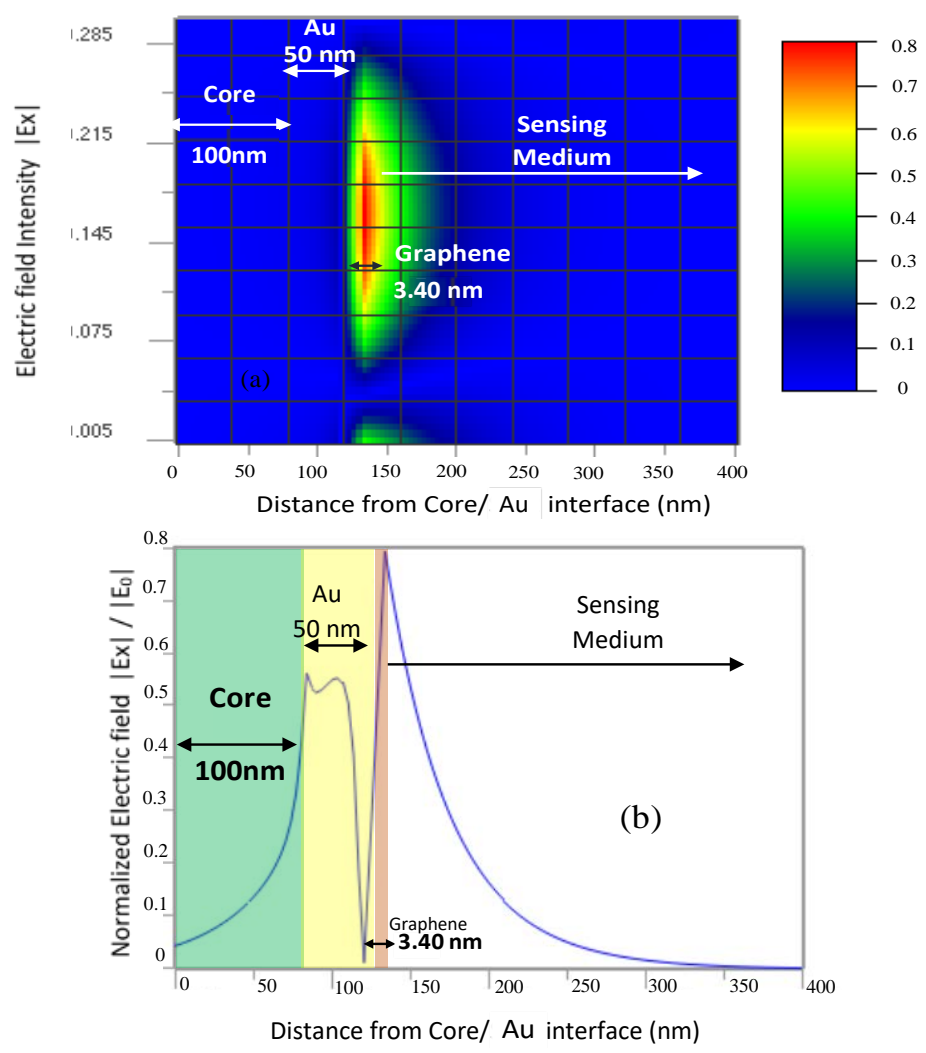

Fig 4. (a) The simulated electric field distribution (b) The electric field intensity with respect to L=10 graphene layers. 
The numerical values of the electric field distribution in the configuration with graphene is greater due to greater absorption of light. Therefore, SPW excitations are stronger for the configuration of Au-Graphene. This proposed optical biosensor is designed and simulated based on SPR technology and SPWs is depends on the variation of the refractive index in the sensing medium.

The addition of 2D material (graphene) over the gold layer can affect the field intensity which causes sensitivity enhancement by increasing the mobility of electrons as depicted in Fig. 5. The electric field intensity approaches to its maximum value when the reflectance curve shows the minimum value of reflectivity. In this case, the highest excitation of SPs can occur. As it can be clearly seen from Fig. 4, the graphene layer increases the field intensity and displays a peak at the gold/sensing medium interface which leads to the excitation of SPs at this interface. The $\mathrm{RI}$ is changed near the graphene interface due to the adsorption of the DNA molecule. The RI of sensing medium has the momentous influence on the reflection power and the SPR curve [4].

Now we investigated the numerical sensitivity which is defined as a function of the change of SPR angle due to the change of refractive index of the sensing medium, so, Sensitivity $(S)=\Delta \theta_{\mathrm{SPR}} / \Delta \mathrm{n}_{\mathrm{s}}[14]$.

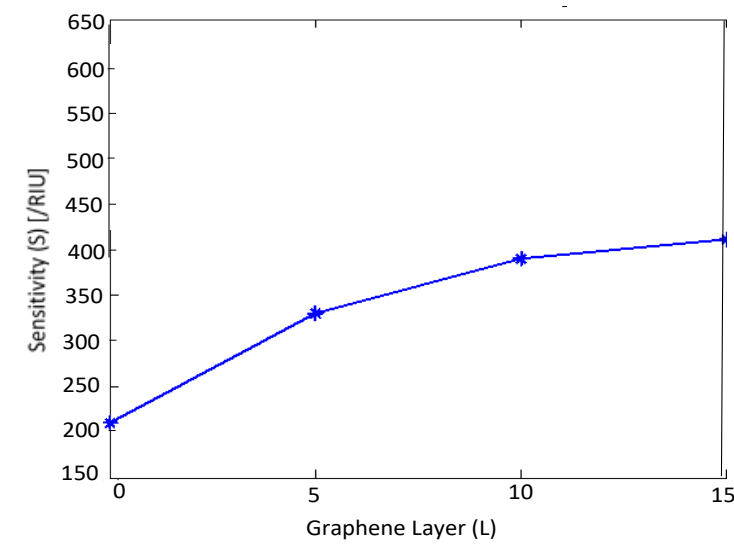

Fig.5. Sensitivity Enhancement curve due to the addition of graphene layers.

It is easily seen from Fig. 5, that sensitivity increases with the increase of graphene layers. In this study, the sensitivity with 10 graphene layers will be improved up to approximately 400\% than bare graphene layer. Owing to increased adsorption capability of DNA molecules on graphene, sensitivity increases compared to the conventional gold thin film SPR biosensor. So, the addition of $\mathrm{N}$ graphene layers is able to enhance the sensitivity by $(1+0.4 \mathrm{~N}) \% \mathrm{RIU}^{-1}$ times.

\section{Conclusion}

We have presented a high-sensitivity fiber optic-based graphene-coated SPR biosensor for sensing DNA hybridization. From the variation of the SPR angle, the proposed sensor can differentiate between complementary and single-base-mismatched DNA. Simulation results indicate that use of graphene can be more effective compared with the usual SPR biosensors. It shows high sensitivity of $40 \%$ / RIU in the case of one layer of graphene-coated sensor. This increased sensitivity is due to the absorption ability, enhancement of the carrier mobility and effective light absorption ability of graphene. At the end of this paper, the electric field distribution study of the proposed work is discussed.

\section{References}

[1] Hossain, Md Biplob and Rana, M. M. Graphene Coated High Sensitive Surface Plasmon Resonance Biosensor for Sensing DNA Hybridization. sensor letters. 2015(13): 1-8.

[2] Hossain, Md Biplob and Rana, M. M. DNA Hybridization Detection based on Resonance Frequency Readout in Graphene on Au SPR Biosensor. Journal of Sensors. 2016.

[3] Shushama, K. N., Rana, M. M., Inum, R., and Hossain, M. B. Graphene coated fiber optic surface plasmon resonance biosensor for the DNA hybridization detection: Simulation analysis. Optics Communications. 2017(383):186-190.

[4] Rahman, M. S., Anower, M. S., Hasan, M. R., Hossain, M. B. and Haque, M. I. Design and numerical analysis of highly sensitive Au-MoS2-graphene based hybrid surface plasmon resonance biosensor. Optics Communications. 2017(396): 36-43. 
[5] Erickson, D., Mandal, S., Yang, A. H. J. and Cordovez, B. Nanobiosensors: optofluidic, electrical and mechanical approaches to biomolecular detection at the nanoscale. Microfluidics and Nanofluidics. 2017(4): 33-52.

[6] Luff, B. J., Wilkinson, J. S., Piehler, J., Hollenbach, U., Ingenhoff, J. and Fabricius, N. Integrated Optical Mach-Zehnder Biosensor. Journal of Lightwave Technology. 1998(16): 583-592.

[7] Altintas, Z., Uludag, Y., Gurbuz, Y. and Tothill, I. E. Surface plasmon resonance based immunosensor for the detection of the cancer biomarker carcinoembryonic antigen. Talanta. 2011(86): 377-383.

[8] Kretschmann, E., \& Raether, H. Notizen: Radiative Decay of Non Radiative Surface Plasmons Excited by Light. Zeitschrift Für Naturforschung A. 2014(23): 2135-2136.

[9] Otto, A. Excitation of nonradiative surface plasma waves in silver by the method of frustrated total reflection. Zeitschrift Für Physik A Hadrons and Nuclei. 1968(216): 398-410.

[10] Wu, L., Chu, H. S., Koh, W. S., \& Li, E. P. Highly sensitive graphene biosensors based on surface plasmon resonance. Optics Express. 2010(1814): 14395-14400.

[11] Rahman, M. S., Anower, M. S., Rahman, M. K., Hasan, M. R., Hossain, M. B. and Haque, M. I. Modeling of a highly sensitive MoS2-Graphene hybrid based fiber optic SPR biosensor for sensing DNA hybridization. Optik - International Journal for Light and Electron Optics. 2017(140): 989-997.

[12] Hossain, M. B., Muktadhir, S. and Rana, M. M. Multi-structural optical devices modeling using graphene trilayer sheets. Optik - International Journal for Light and Electron Optics. 2016(127): 5841-5851.

[13] Rong, G., Najmaie, A., Sipe, J. E. and Weiss, S. M. Porous Silicon Waveguides for DNA Detection. In: 3rd IEEE International Conference on Group IV Photonics; 2006.p. 13-15.

[14] G. Eda and S. A. Maier. Two-Dimensional Crystals: Managing Light for Optoelectronics. ACS Nano. 2018(7): 5660- 5665.

[15] J. Majka and C. Speck. Analysis of protein-DNA interactions using surface plasmon resonance. Advances in Biochemical Engineering/Biotechnology. 2007(104): 13-36.

[16] Vasilescu, A., Gáspár, S., Gheorghiu, M., David, S., Dinca, V., Peteu, S. and Szunerits, S. Surface Plasmon Resonance based sensing of lysozyme in serum on Micrococcus lysodeikticus-modified graphene oxide surfaces. Biosensors and Bioelectronics. 2017(89): 525-531.

[17] M.S. Rahman, M.R. Hasan, K.A. Rikta and M.S. Anower. A novel graphene coated surface plasmon resonance biosensor with tungsten disulfide (WS2) for sensing DNA hybridization. Opt. Mater. 2018(75): 567-573.

[18] Ishimaru, A. Electromagnetic Wave Propagation, Radiation, and Scattering: From Fundamentals to Applications. John Wiley \& Sons. Prentice Hall, Upper Saddle River, NJ, USA; 1991.

[19] V. Ball and J. J. Ramsden. Buffer dependence of refractive index increments of protein solutions. Biopolymers. 1998(46): 489-492.

(C) 2019 by the author(s). This work is licensed under a Creative Commons Attribution 4.0 International License (http://creativecommons.org/licenses/by/4.0/). Authors retain copyright of their work, with first publication rights granted to Tech Reviews Ltd. 